\title{
Cortical Excitability and Connectivity in Patients With Brain Tumors
}

\author{
Vincenzo Rizzo ${ }^{1 *}$, Carmen Terranova ${ }^{1}$, Giovanni Raffa ${ }^{2}$, Salvatore Massimiliano Cardali ${ }^{2}$, \\ Filippo Flavio Angileri ${ }^{2}$, Giuseppina Marzano ${ }^{1}$, Maria Catena Quattropani ${ }^{1}$, \\ Antonino Germanò ${ }^{2}$, Paolo Girlanda ${ }^{1}$ and Angelo Quartarone ${ }^{3}$
}

${ }^{1}$ Department of Clinical and Experimental Medicine, University of Messina, Messina, Italy, ${ }^{2}$ Division of Neurosurgery, BIOMORF Department, University of Messina, Messina, Italy, ${ }^{3}$ Department of Biomedical Science and Morphological and Functional Images, University of Messina, Messina, Italy

\section{OPEN ACCESS}

Edited by:

Pierpaolo Peruzzi,

Brigham and Women's Hospital and Harvard Medical School,

United States

Reviewed by:

Claudia Katharina Petritsch Stanford University, United States Tomofumi Yamaguchi, Juntendo University, Japan

${ }^{*}$ Correspondence: Vincenzo Rizzo

enzo.rizzo@gmail.com

Specialty section:

This article was submitted to Neuro-Oncology and Neurosurgical Oncology, a section of the journal Frontiers in Neurology

Received: 28 February 2021 Accepted: 29 June 2021

Published: 26 August 2021

Citation:

Rizzo V, Terranova C, Raffa G, Cardali SM, Angileri FF, Marzano G,

Quattropani MC, Germanò A, Girlanda P and Quartarone A (2021)

Cortical Excitability and Connectivity in

Patients With Brain Tumors.

Front. Neurol. 12:673836

doi: 10.3389/fneur.2021.673836
Background: Brain tumors can cause different changes in excitation and inhibition at the neuronal network level. These changes can be generated from mechanical and cellular alterations, often manifesting clinically as seizures.

Objective/Hypothesis: The effects of brain tumors on cortical excitability (CE) have not yet been well-evaluated. The aim of the current study was to further investigate cortical-cortical and cortical-spinal excitability in patients with brain tumors using a more extensive transcranial magnetic stimulation protocol.

Methods: We evaluated CE on 12 consecutive patients with lesions within or close to the precentral gyrus, as well as in the subcortical white matter motor pathways. We assessed resting and active motor threshold, short-latency intracortical inhibition (SICI), intracortical facilitation (ICF), short-latency afferent inhibition (SAI), long-latency afferent inhibition, cortical silent period, and interhemispheric inhibition.

Results: CE was reduced in patients with brain tumors than in healthy controls. In addition, SICI, ICF, and SAI were lower in the affected hemisphere compared to the unaffected and healthy controls.

Conclusions: $C E$ is abnormal in hemispheres affected by brain tumors. Further studies are needed to determine if $\mathrm{CE}$ is related with motor impairment.

Keywords: cortical excitability, brain tumors, transcranial magnetic stimulation, motor function, recovery

\section{INTRODUCTION}

The "maximal safe resection" represents the goal standard of the modern surgical treatment of brain tumors located in eloquent areas. Various techniques supply important anatomical and functional information regarding the brain functional organization. Different neuroimaging and neurophysiological techniques can be used to plan a surgical strategy to preserve functional networks and to increase the maximal safe resection. Navigated transcranial magnetic stimulation (nTMS) is a helpful tool for preoperative cortical mapping and planning before surgery of brain tumors located in eloquent areas (1-5). Brain tumors like brain trauma, hematoma, and focal cerebral ischemia can cause brain parenchyma compression that can produce changes in excitation and inhibition, even in the absence of histologically significant cell injury, often manifesting clinically as seizures. The precise mechanism producing seizures after cortical compression remains elusive (6). Recent studies used preoperative nTMS as a 
predictor of motor outcome in patients with brain tumors. Rosenstock et al. showed that an abnormal interhemispheric resting motor threshold (RMT) ratio was related to a higher risk for poor postoperative outcome in the 1st week, but not in the following 3 months (7). This proposed stratification model, based on functional-anatomical and neurophysiological measures, could allow quantification of the functional impairment or recovery potential. Several parameters of cortical excitability have been studied in patients with traumatic brain injury (8) and stroke (9). In a recent paper, Neville et al. (10) described an increase in motor threshold (MT) that was paralleled by an alteration in short-latency intracortical inhibition (SICI) and intracortical facilitation (ICF) (10). However, the authors did not check other important parameters of cortical excitability such as short-latency afferent inhibition (SAI), long-latency afferent inhibition (LAI), cortical silent period (CSP), recruitment curve (RC), and interhemispheric inhibition (IHI). The aim of the current study was to further investigate cortical-cortical and cortical-spinal excitability in patients with brain tumors using a more extensive TMS protocol.

\section{MATERIALS AND METHODS}

The present study was conducted in accordance with the ethics committee of the University of Messina and the Declaration of Helsinki. Informed consent was obtained from every patient.

\section{Patient Population}

The cortical excitability measurements by transcranial magnetic stimulation (TMS) were carried out on 12 consecutive patients with lesions involving the primary motor cortex (M1) and corticospinal tract (CST). Not all 12 patients participated in all cortical excitability measurements (see below). Table 1 reports all nosographic data and neurological status of patients. Exclusion criteria for brain stimulation were the same as for magnetic resonance imaging (MRI). Patients were examined for handedness, motor impairment, medical history, and use of medication (see Table 1). The WHO classification was used for tumor histology (11).

\section{Measures of Cortical Excitability}

Patients and controls were seated in a "comfortable reclining chair and surface EMG was recorded from the right or left first dorsal interosseus (FDI) muscle using disposable disc electrodes with a belly-tendon montage. EMG was filtered by Neurolog System supplied by Digitimer with a time constant of $3 \mathrm{~ms}$, and a high pass filter set a $3 \mathrm{kHz}$." Single or paired pulses were given to the right or left M1 using a standard figure-ofeight coil connected with a single (for single-pulse TMS) or two (for paired-pulse TMS) high-power Magstim 200 stimulators. "Signals were collected via a CED 1401 laboratory interface (Cambridge Electronic Design, Cambridge, UK) and fed to a personal computer for offline analysis" (12).

\section{Threshold Measurements}

In 10 patients, we evaluated RMT and active MT (AMT). "RMT was defined as the minimum intensity that evoked a peak-to-peak motor evoked potential (MEP) of $50 \mu \mathrm{V}$ in at least 5 out of 10 consecutive trials in the relaxed FDI muscle. AMT was defined as the minimum intensity that elicited a reproducible MEP of at least $200 \mu \mathrm{V}$ in the tonically contracting FDI muscle in at least 5 out of 10 consecutive trials" (13).

\section{Recruitment Curve}

In 12 patients, we evaluated input-output RC. "Motor evoked potentials (MEPs) input-output recruitment curve was performed at stimulus intensities ranging from 100 to $150 \%$ RMT (in steps of 10\%). Fifteen peak-to-peak MEP at each stimulation intensities were averaged" (12).

\section{Intracortical Paired-Pulse Excitability}

In 10 patients, we studied SICI and ICF. SICI and ICF were determined according to the paired-pulse method described by Kujirai et al. (14). The intensity of the conditioning stimulus was set at $80 \%$ of AMT, while the test stimulus was adjusted to elicit MEPs with amplitudes of $0.5-1.0 \mathrm{mV}$ at baseline (115$125 \%$ of RMT in healthy subjects, and $\sim 140-150 \%$ of the RMT in patients with brain tumors). SICI and ICF were assessed at ISIs of 2 and $12 \mathrm{~ms}$, respectively. The mean amplitude of the conditioned MEP was expressed as percentage of the amplitude of the unconditioned MEP. This characterized the strength of SICI and ICF.

\section{Cortical Silent Period}

In eight patients, we evaluated CSP. CSP was measured during slight tonic contraction of the right or left FDI muscle at $\sim 10-$ $15 \%$ of maximum force level measurements. The intensity of the test stimulus was $130 \%$ of resting MT. The duration of the CSP was measured in each trial (15).

\section{Sensorimotor Intracortical Inhibition}

In nine patients, we studied SAI and LAI, which were studied using the conditioning test protocol described by Tokimura et al. (16). The median nerve was stimulated through bipolar electrodes at the wrist (cathode proximal). The intensity was set just approximately three times the perceptual threshold. The intensity of the transcranial test stimulus was adjusted to evoke a muscle response in relaxed abductor pollicis brevis (APB) with a peak-to-peak amplitude of $\sim 0.5-1 \mathrm{mV}$ (115-125\% of RMT in healthy subjects, and $\sim 140-150 \%$ of RMT in patients with brain tumors). SAI and LAI were probed at ISIs of 20, 25, and $200 \mathrm{ms,}$ respectively. The relative change in MEP amplitude induced by the peripheral stimulus was taken as a measure of SAI and LAI.

\section{Interhemispheric Inhibition}

"A conditioning-test protocol as described by Ferbert et al. (17) was used to evaluate IHI of the right or left M1. IHI was studied in 8 patients. A conditioning stimulus was applied to the left or right M1, and the test stimulus was applied to the homologous right or left M1. We set the intensity of the first (conditioning) stimulus to obtain an inhibition of the test MEP to about $50 \%$ at an ISI of $10 \mathrm{~ms}$. The second (test) stimulus was set at an intensity that, when given alone, would evoke an EMG response of $0.5-1 \mathrm{mV}$ peak-to-peak amplitude (115-125\% of RMT in healthy subjects, 
TABLE 1 | Summary of patients' epidemiological data.

\begin{tabular}{|c|c|c|c|c|c|c|}
\hline & Age, sex & Handedness & Tumor location & Neurological examination & Histology & AEDs \\
\hline \#1, DM & $51, F$ & $\mathrm{R}$ & $\mathrm{MC} \mathrm{I}$, right & $\begin{array}{l}\text { Moderate left upper limb } \\
\text { weakness }\end{array}$ & $\begin{array}{l}\text { Oligodendroglioma } \\
\text { IDH-mutant } \\
\text { 1p/19q-codeleted }\end{array}$ & LEV \\
\hline$\# 2, \mathrm{BA}$ & $38, M$ & $\mathrm{R}$ & MC I left & No deficit & $\begin{array}{l}\text { Oligodendroglioma } \\
\text { IDH-mutant } \\
\text { 1p/19q-codeleted }\end{array}$ & $/ /$ \\
\hline$\# 3, \mathrm{FT}$ & $69, F$ & $\mathrm{R}$ & Fronto-temporo-insular, left & No deficit & Glioblastoma IDH-wildtype & $/ /$ \\
\hline$\# 4$, CS & $35, M$ & $\mathrm{R}$ & Fronto-temporo-insular, right & No deficit & $\begin{array}{l}\text { Diffuse astrocytoma } \\
\text { IDH-wildtype }\end{array}$ & LEV \\
\hline \#5, CG & $46, M$ & $\mathrm{R}$ & Fronto-insular, left & No deficit & Glioblastoma IDH-mutant & $/ /$ \\
\hline \#6, CT & $70, F$ & $\mathrm{R}$ & Fronto-opercular, left & No deficit & Gliobastoma IDH-wildtype & LEV \\
\hline$\# 7$, VA & $67, M$ & $\mathrm{R}$ & Fronto-temporal, right & No deficit & Gliobastoma IDH-wildtype & $/ /$ \\
\hline \#8, CG & $46, M$ & $\mathrm{~L}$ & Fronto-temporal, right & No deficit & $\begin{array}{l}\text { Diffuse astrocytoma } \\
\text { IDH-wildtype }\end{array}$ & $/ /$ \\
\hline$\# 9, \mathrm{PN}$ & $53, F$ & $\mathrm{R}$ & Fronto-temporal, right & No deficit & Oligodendroglioma NOS & // \\
\hline \#10, MR & $60, F$ & $\mathrm{R}$ & Temporo-parietal, left & No deficit & Gliobastoma IDH-wildtype & LEV \\
\hline \#11, MW & $36, M$ & $\mathrm{~L}$ & Fronto-temporo-insular, right & No deficit & $\begin{array}{l}\text { Diffuse astrocytoma } \\
\text { IDH-wildtype }\end{array}$ & LEV \\
\hline \#12, BAG & $55, \mathrm{M}$ & $\mathrm{R}$ & Temporo-parietal, right & No deficit & Gliobastoma IDH-wildtype & LEV \\
\hline
\end{tabular}

Nosographic data and neurological status for all 12 consecutive patients with lesions within or close to the precentral gyrus. MC, motor cortex; LEV, levetiracetam.

$\sim 140-150 \%$ of the RMT in patients with brain tumors). IHI was tested at three conditioning-test intervals (8, 9, $10 \mathrm{~ms})$ " (18).

\section{Statistical Analysis}

Factorial ANOVA was computed to show differences in RMT; SAI 20 and $25 \mathrm{~ms}$; LAI; SICI; ICF; IHI at 8, 9, and $10 \mathrm{~ms}$; and CSP between the affected hemisphere, unaffected hemisphere, and controls. MEP RCs were evaluated in separate repeatedmeasures ANOVA in the different sets of subjects. We performed a two-way repeated-measures ANOVA with intensity (six levels: $100,110,120,130,140,150 \%$ of MT) as within-subject factor, and group (three levels: affected hemisphere, unaffected hemisphere, and controls) as between-subjects factor. If appropriate, post hoc $t$-tests were performed. Post hoc Fisher's PLSD analysis was executed for RC. Significance was set at $p<0.05$. Data are given as mean \pm standard error of the mean.

\section{RESULTS}

No participants reported any adverse effects during or after the study.

\section{Motor Threshold}

RMT and AMT were significantly higher in patients than in controls $\left[\mathrm{RMT}: F_{(2,26)}=4.03, p=0.029\right.$; power $=0.66$; AMT: $F_{(2,26)}=4.1, p=0.028$, power $\left.=0.65\right]$ (Figure 1). Post hoc $t$-tests revealed relative change only between controls and the affected hemisphere of patients [for RMT: $t_{(1,9)}=2.6, p=0.017$; for AMT: $\left.t_{(1,9)}=2.2, p=0.03\right]$.

\section{Recruitment Curve}

MEP amplitudes increased with increasing stimulus intensity in controls and patients. However, MEP RC was significantly less steep in patients in both hemispheres compared to controls (Figure 2). Indeed, repeated ANOVA indicated a significant effect for intensity $\left[F_{(2,29)}=50.9, p<0.0001\right.$; power $\left.=1.0\right]$ with a significant interaction between intensity and groups $\left[F_{(2,29)}=\right.$ $18.780, p<0.0001$; power $=1.0]$. Post hoc Fisher's PLSD analysis showed that MEP amplitudes were significantly higher in control subjects compared to the affected $(p<0.001)$ and unaffected hemispheres of patients $(p<0.001)$. On the contrary, there were no differences between the RCs of both affected and unaffected hemispheres $(p=0.9)$ of patients.

\section{Intracortical Paired-Pulse Excitability}

Paired-pulse stimulation consistently produced SICI at an ISI of $2 \mathrm{~ms}$ and ICF at an interval of $12 \mathrm{~ms}$ in controls, but not so well in patients. The data showed a lower degree of inhibition and facilitation in brain tumor patients (Figure 3). ANOVA showed a main effect between patients (affected and unaffected hemispheres) and controls for ICI $\left[F_{(2,27)}=3.87, p=0.03\right.$; power $=0.65]$ and ICF $\left[F_{(2,27)}=3.58, p=0.042\right.$; power $\left.=0.58\right]$. Post hoc $t$-tests revealed relative change only between controls and affected hemisphere of patients for ICI $\left[t_{(1,9)}=4.10, p=0.0006\right]$ and ICF $\left[t_{(1,9)}=2.13, p=0.046\right]$, but none between controls and unaffected hemisphere of patients [ICI: $t_{(1,9)}=1.8, p=0.08$; ICF: $\left.t_{(1,9)}=1.7, p=0.1\right]$.

\section{Sensorimotor Intracortical Inhibition}

ANOVA showed a selective reduction in SAI (20 and $25 \mathrm{~ms}$ ) but not in LAI $(200 \mathrm{~ms}$ ) (see Figure 4). For SAI, there was a 


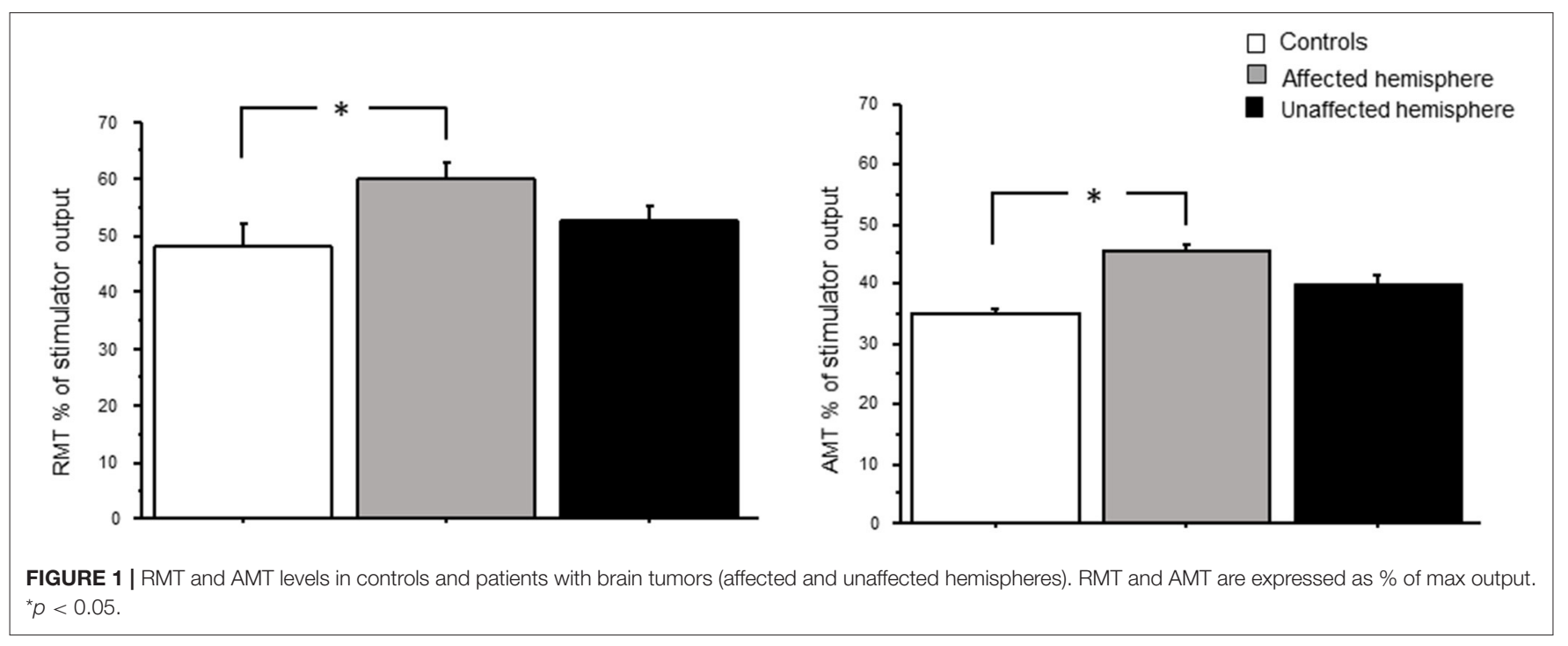

FIGURE 1 | RMT and AMT levels in controls and patients with brain tumors (affected and unaffected hemispheres). RMT and AMT are expressed as \% of max output.
${ }^{*} p<0.05$.

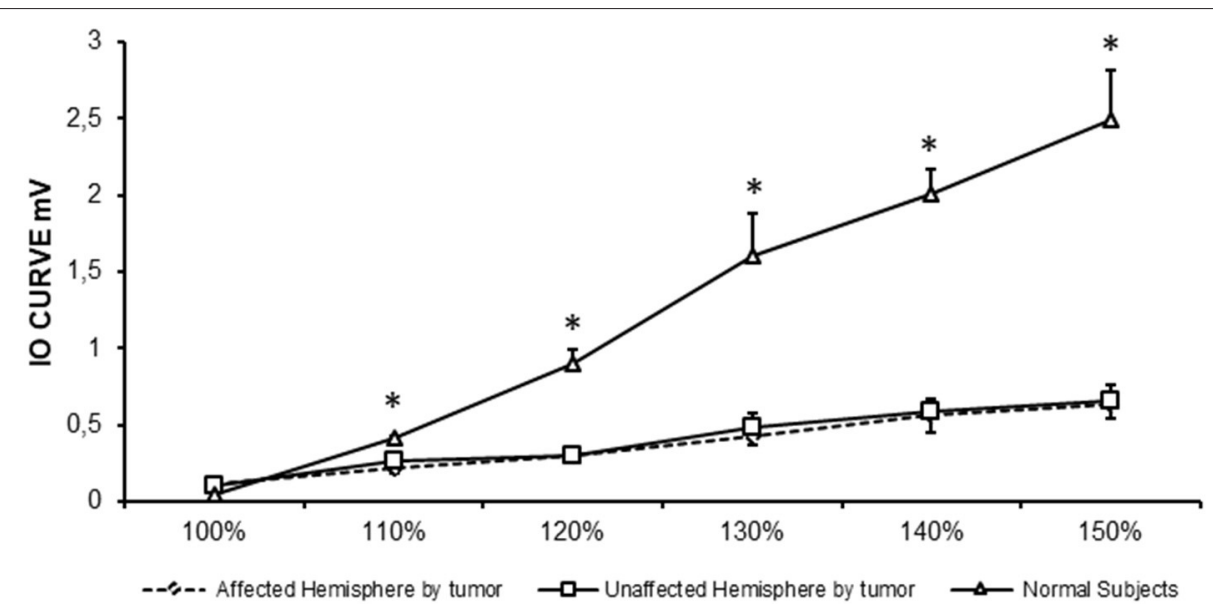

FIGURE 2 | RC in controls and patients with brain tumors (affected and unaffected hemispheres). Stimulus intensities ranging from 100 to $150 \%$ RMT (in steps of $10 \%) .{ }^{*} p<0.05$.

prominent main effect for ISI at $25 \mathrm{~ms}\left[F_{(2,29)}=5.33 ; p=0.01\right.$; power $=0.8]$ and $20 \mathrm{~ms}\left[F_{(2,29)}=4.29 ; p=0.02\right.$; power $\left.=0.7\right]$. Post hoc $t$-tests revealed significant change only between controls and affected hemisphere of patients for SAI at $25 \mathrm{~ms}\left[t_{(1,9)}=3.6\right.$, $p=0.0015]$ and $20 \mathrm{~ms}\left[t_{(1,9)}=2.7, p=0.011\right]$. For LAI, ANOVA demonstrated no main effect $\left[F_{(2,29)}=2.7 ; p=0.08\right]$.

\section{Cortical Silent Period}

The duration of CSP did not differ between patients (affected and unaffected hemispheres) and controls $\left[F_{(2,21)}=0.75 ; p=0.48\right]$ (see Figure 5).

\section{Interhemispheric Inhibition}

Figure 5 shows also the time course of IHI in patients (affected and unaffected hemispheres) and controls. Repeated-measures ANOVA did not reveal a significant interaction between the two main factors of ISI and population for no interval $\left[8 \mathrm{~ms}: F_{(2,23)}=\right.$
$3.17 ; p=0.07 ; 9 \mathrm{~ms}: F_{(2,23)}=2.34 ; p=0.11 ; 10 \mathrm{~ms}: F_{(2,23)}=2.82$; $p=0.08]$.

\section{DISCUSSION}

Our data yielded three main findings. First, affected and unaffected hemisphere excitability in patients with brain tumors was reduced compared to healthy controls. Second, SICI, ICF, and SAI were lower in the affected hemisphere. Third, IHI, LAI, and CSP showed no differences between patients and healthy controls. These findings indicate that the effects of brain tumors on cortical excitability are mostly localized to the affected hemisphere.

\section{Effects on Corticospinal Excitability}

MT is an indicator of cortical excitability reflecting membrane excitability (19). In our study, RMT and AMT were significantly 

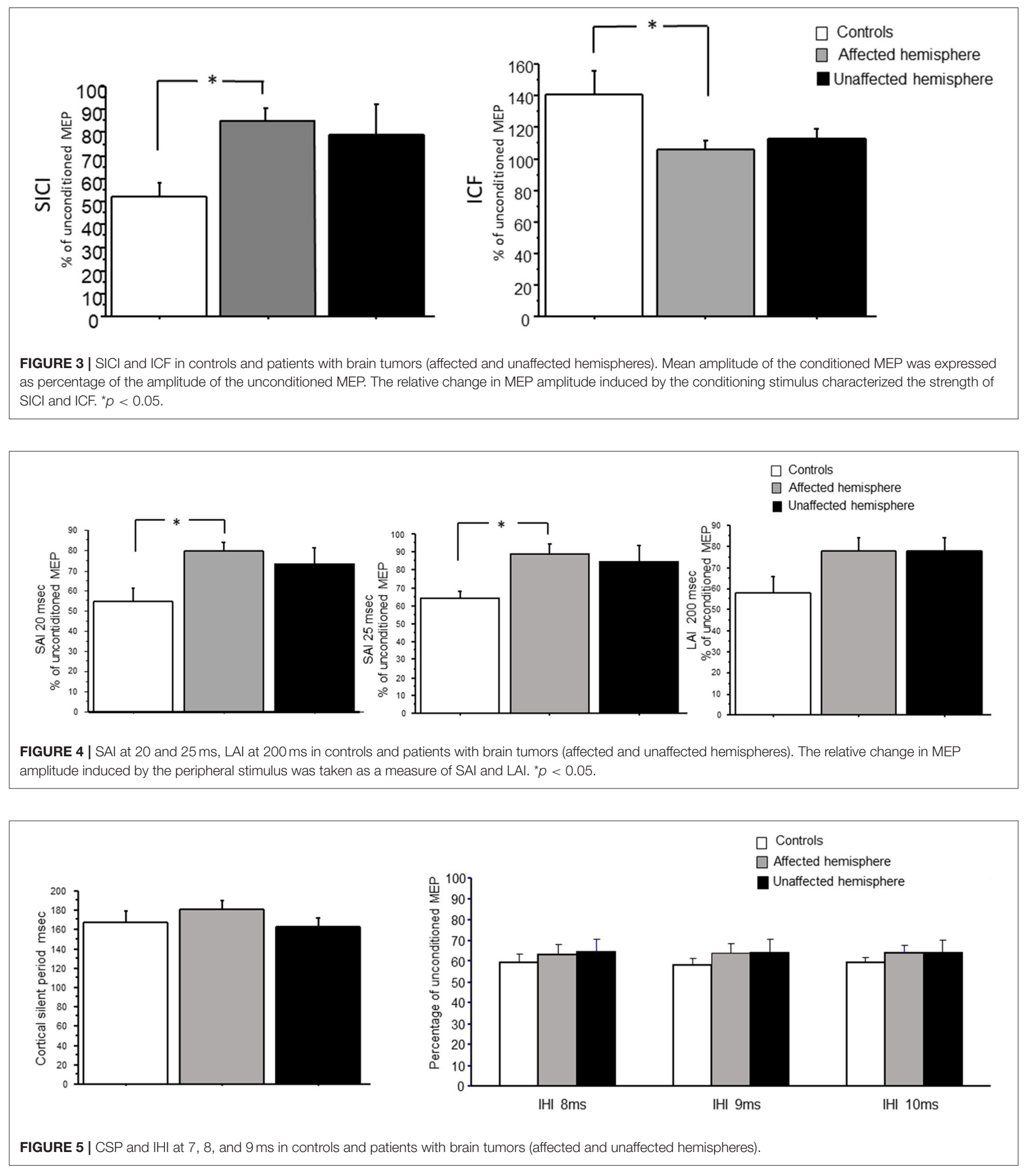

higher in patients, especially in the affected hemisphere, than in healthy controls. This result may be due to a reduced density and number of corticospinal neurons in relation to motor impairment. However, in our study, only one patient had a moderate left upper limb weakness. In addition, higher MT may predict a poor motor outcome in patients with brain tumors (20). Picht et al. speculated that patients initially without hemiparesis but with high RMT were at a higher risk in the 
long term of a decline in motor function (20). Rosenstock et al. studied abnormal RMT interhemispheric ratio was related to a higher risk for poor postoperative outcome in the 1st week, but not in the following 3 months (7). Similar to previous findings, Neville et al. reported an increase in MT in patients with brain tumors (10). Furthermore, in stroke patients, Swayne et al. (21) demonstrated that corticospinal excitability of the affected hemisphere, measured as AMT and RMT, increased in the acute phase, but this increment became weaker at 3 months and it continued for 6 months (chronic phase of stroke). The authors concluded that, in the chronic phase of stroke, the motor function could be dependent on the reorganization of alternative cortical networks (21). Moreover, our data show that MEP RC was significantly less steep in patients in both hemispheres compared to controls. RC illustrates a graded profile of cortical spinal tract (CST) function, providing a more global measure of cortical excitability than MT (22). Alterations in the slope of the RC can predict more substantial CST damage, motor impairment, and poor recovery in patients with brain injury (23). RCs are widely used in stroke because they are believed to reflect CST gain and output from the primary motor cortex (24). In our study, MEP RC was significantly less steep in patients in both hemispheres compared to controls. Experimental data in animal model suggest that glioma cells release high amounts of glutamate resulting in excitotoxicity and tumor invasion $(25,26)$. Therefore, it is likely that excitotoxicity at a chronic stage may result in a significant loss of motor neurons within primary motor area and a loss of CSTs as indexed by the increase in RMT and AMT and by the reduced slope of RC. The excitotoxicity promoted by infiltrating glioma cells may also in turn affect fast-spiking $\mathrm{GABA}_{\mathrm{A}}$ interneurons, and a reduced GABA availability may create a vicious circle where increased pyramidal neurons firing amplify glutamate excitotoxicity (27), producing neuronal cell death (see below).

\section{Effects on Cortical Excitability}

We showed that SICI, ICF, and SAI are reduced in the affected hemisphere compared to the unaffected and healthy controls. On the other hand, there were no significant differences of LAI and CSP between patients and healthy controls. SICI is mediated by $\mathrm{GABA}_{\mathrm{A}}$ receptors, while CSP is a marker for the excitability of long-lasting (presumably $G_{A B A}$ ) intracortical interneurons. Conversely, ICF is mediated by glutamate and is associated with excitatory cortical circuits (28-30). In a case report, only the lack of inhibition, assessed by SICI, had been demonstrated in two patients with focal motor seizures caused by a circumscribed glioblastoma or metastasis (31). The results of our study are in agreement with similar findings of Neville et al., who reported an alteration of SICI and ICF in the affected hemisphere (10). The novel finding of a reduced SICI and ICF in the affected hemisphere could be caused by the simultaneous selective reduction in GABAergic $\mathrm{A}$ inhibition and glutamatergic excitation because of either reduced excitability or loss of inhibitory/excitatory neurons or changes in GABAergic/glutamatergic receptor function. The presence of a disruption of $\mathrm{GABA}_{\mathrm{A}}$ mechanisms in the affected hemisphere could explain the high percentage of seizures (50\%) in our population. On the other hand, this disinhibition could also be an adaptive plastic mechanism recruited by the affected hemisphere to counteract the reduced overall excitability caused by tumorrelated brain edema and swelling (see above). These findings parallel evidences in stroke patients where SICI is reduced in the affected hemisphere in the first 6 months with normal CSP and may contribute to cortical reorganization and recovery (32).

This reduced $\mathrm{GABA}_{\mathrm{A}}$ intracortical inhibition is in line with several findings coming from the bench side. The largest class (40-50\%) of GABAergic interneurons is represented by fastspiking, parvalbumin-positive cells, which are present in all layers and form synapses on the soma and proximal dendrites of pyramidal cells (33). Several pieces of evidence in animal models have demonstrated a loss of fast-spiking interneurons, and more in general a reduced firing rate in the peritumoral glioma area (34). This dysfunction of fast-spiking GABAa interneurons is critically involved in tumor-associated epileptic seizures (34).

Interestingly, it has been demonstrated that the selective optogenetic stimulation of parvalbumin-positive $\mathrm{GABA}_{\mathrm{A}}$ interneurons induces a significant reduction in glioma cell proliferation (27). Human glioblastoma cells may express functional $\mathrm{GABA}_{\mathrm{A}}$ receptors, and that endogenous GABA release may attenuate tumor proliferation (35). On the other hand, pyramidal cell stimulation enhances cell proliferation in tumor mass (36). It is likely that fast-spiking $\mathrm{GABA}_{\mathrm{A}}$ interneuron vulnerability to tumor-induced excitotoxicity may trigger a vicious circle where reduced GABA availability may increase pyramidal neuron firing, producing an enhanced tumor growth. Future studies in humans are needed to better understand the relationship of SICI with tumor proliferation.

Another important finding was a selective reduction in SAI $(20$ and $25 \mathrm{~ms})$ but not in LAI $(200 \mathrm{~ms})$ in the affected hemisphere. LAI and SAI are mediated through different sensorimotor circuits. SAI is controlled by muscarinic neurotransmission (37). LAI is significantly understudied compared to SAI, and the neural circuitries underlying these phenomena are unclear (38). SAI has been used to assess and predict functional recovery following ischemic stroke, where larger SAI reductions correlate with improved long-term recovery 6 months following injury (39). The presence of a reduced SAI in the affected hemisphere of patients with brain tumor needs to be better investigated in future longitudinal studies, especially in low-grade glioma, to see if SAI reduction is heralding a good recovery after surgical operation.

\section{Effects on IHI}

Our study demonstrates no differences in IHI between patients and controls. The normalcy of IHI confirms previous evidence suggesting that a physiological interplay between the two primary motor cortices is required to maintain a good motor function in patients with brain tumor (40). In a very elegant study, brain connectivity was measured in patients with glioma using task-free functional MRI to probe motor networks. Patients with motor weakness showed reduced interhemispheric and left primary motor cortex and the right premotor area connectivity compared to healthy controls. Conversely, in patients without motor deficit, motor performance assessed on the grooved 
pegboard was not related to interhemispheric connectivity, which was unchanged but correlated with ipsilateral connectivity between the premotor area and supplementary motor area (40).

The absence of motor impairment in our cohort of patients could be explained by the normalcy of transcallosal connection evaluated using the IHI. Indeed, Otten et al. showed that an integrity of transcallosal pathway between the two motor areas is needed to maintain a normal motor function (40). Since TMS is a non-invasive technique, future longitudinal studies are warranted to explore the role of IHI to predict motor outcome in patients with brain tumor.

\section{CONCLUSIONS}

This study investigates cortical-cortical and cortical-spinal excitability in patients with brain tumors using a more extensive TMS protocol. Different measurements of cortical excitability are abnormal in brain hemispheres affected by tumors, but further studies are needed to determine their relationship to motor impairment and subsequent motor recovery. Finally, it will be important to explore the correlation between brain tumor molecular features and the impairment of cortical excitability in a lager sample population.

\section{REFERENCES}

1. Hervey-Jumper SL, Berger MS. Role of surgical resection in low- and high-grade gliomas. Curr Treat Options Neurol. (2014) 16:284. doi: 10.1007/s11940-014-0284-7

2. Rizzo V, Terranova C, Crupi D, Sant'angelo A, Girlanda P, Quartarone A. Increased transcranial direct current stimulation after effects during concurrent peripheral electrical nerve stimulation. Brain Stimul. (2014) 7:113-21. doi: 10.1016/j.brs.2013.10.002

3. Raffa G, Scibilia A, Conti A, Ricciardo G, Rizzo V, Morelli A, et al. The role of navigated transcranial magnetic stimulation for surgery of motor-eloquent brain tumors: a systematic review and metaanalysis. Clin Neurol Neurosurg. (2019) 180:7-17. doi: 10.1016/j.clineuro.2019.03.003

4. Raffa G, Scibilia A, Conti A, Cardali SM, Rizzo V, Terranova C, et al. Multimodal surgical treatment of high grade gliomas in the motor area: the impact of the combination of navigated transcranial magnetic stimulation and fluorescein-guided resection. World Neurosurg. (2019) 128:e37890. doi: 10.1016/j.wneu.2019.04.158

5. Raffa G, Quattropani MC, German,ò A. When imaging meets neurophysiology: the value of navigated transcranial magnetic stimulation for preoperative neurophysiological mapping prior to brain tumor surgery. Neurosurg Focus. (2019) 47:E10. doi: 10.3171/2019.9.FOCUS19640

6. Ming-Chieh D, Wang Q, Lo EH, Stanley GB. Cortical excitation and inhibition following focal traumatic brain injury. J Neurosci. (2011) 31:1408594. doi: 10.1523/JNEUROSCI.3572-11.2011

7. Rosenstock T, Grittner U, Acker G, Schwarzer V, Kulchytska N, Vajkoczy $\mathrm{P}$, et al. Risk stratification in motor area-related glioma surgery based on navigated transcranial magnetic stimulation data. Neurosurg. (2017) 126:1227-37. doi: 10.3171/2016.4.JNS152896

8. Hayashi CY, Neville IS, Rodrigues PA, Galhardoni R, Brunoni AR, Zaninotto $\mathrm{AL}$, et al. Altered intracortical inhibition in chronic traumatic diffuse axonal injury. Front Neurol. (2018) 9:189. doi: 10.3389/fneur.2018.00189

9. McDonnell MN, Stinear CM. TMS measures of motor cortex function after stroke: A meta-analysis. Brain Stimul. (2017) 10:721-34. doi: 10.1016/j.brs.2017.03.008

10. Neville IS, Gomes Dos Santos A, Almeida CC, Hayashi CY, Solla DJF, Galhardoni R, et al. Evaluation of changes in preoperative cortical excitability

\section{DATA AVAILABILITY STATEMENT}

The raw data supporting the conclusions of this article will be made available by the authors, without undue reservation.

\section{ETHICS STATEMENT}

The studies involving human participants were reviewed and approved by AOU G. Martino. The patients/participants provided their written informed consent to participate in this study.

\section{AUTHOR CONTRIBUTIONS}

CT, GR, GM, SC, and FA contributed to the acquisition, analysis and interpretation of data, and writing of the first draft. VR and AQ were responsible for the conception and design, data acquisition, analysis and interpretation as well as the review, and critique of the manuscript. MQ, PG, and AG contributed to interpretation of data and the review and critique of the final manuscript. All authors contributed to the article and approved the submitted version.

by navigated transcranial magnetic stimulation in patients with brain tumor. Front Neurol. (2021) 11:582262. doi: 10.3389/fneur.2020.582262

11. Louis DN, Perry A, Reifenberger G, von Deimling A, Figarella-Branger D, Cavenee WK, et al. The 2016 world health organization classification of tumors of the central nervous system: a summary. Acta Neuropathol. (2016) 131:803-20. doi: 10.1007/s00401-016-1545-1

12. Rizzo V, Crupi D, Bagnato S, Quartarone A, Benvenga S, Bartolone L, et al. Neural response to transcranial magnetic stimulation in adult hypothyroidism and effect of replacement treatment. J Neurol Sci. (2008) 266:38-43 doi: 10.1016/j.jns.2007.08.031

13. Rizzo V, Terranova C, Conti A, Germanò A., Alafaci C, Raffa G, et al. Preoperative functional mapping for rolandic brain tumor surgery. Neurosci Lett. (2014) 583:136-41. doi: 10.1016/j.neulet.2014.09.017

14. Kujirai T, Caramia MD, Rothwell JC, Day BL, Thompson PD, Ferbert A,Wroe S, et al. Corticocortical inhibition in human motor cortex. J Physiol. (1993) 471:501-19. doi: 10.1113/jphysiol.1993.sp019912

15. Orth M, Rothwell JC. The cortical silent period: intrinsic variability and relation to the waveform of the transcranial magnetic stimulation pulse. Clin Neurophysiol. (2004) 115:1076-82. doi: 10.1016/j.clinph.2003.12.025

16. Tokimura H, Di Lazzaro V, Tokimura Y, Oliviero A, Profice P, Inolsa A, et al. Short latency inhibition of human hand motor cortex by somatosensory input from the hand. J Physiol. (2000) 523:503-13. doi: 10.1111/j.1469-7793.2000.t01-1-00503.x

17. Ferbert A, Priori A, Rothwell JC, Day BL, Colebatch JG, Marsden CD. Interhemispheric inhibition of the human motor cortex. J Physiol. (1992) 453:525-46. doi: 10.1113/jphysiol.1992.sp019243

18. Gilio F, Rizzo V, Siebner HR, Rothwell JC. Effects on the right motor hand-area excitability produced by low-frequency rTMS over human contralateral homologous cortex. J Physiol. (2003) 551:56373. doi: 10.1113/jphysiol.2003.044313

19. Wassermann EM. Variation in the response to transcranial magnetic brain stimulation in the general population. Clin Neurophysiol. (2002) 113:116571. doi: 10.1016/S1388-2457(02)00144-X

20. Picht T, Strack V, Schulz J, Zdunczyk A, Frey D, Schmidt S, et al. Assessing the functional status of the motor system in brain tumor patients using transcranial magnetic stimulation. Acta Neurochir (Wien). (2012) 154:207581. doi: 10.1007/s00701-012-1494-y 
21. Swayne OB, Rothwell JC, Ward NS, Greenwood RJ. Stages of motor output reorganization after hemispheric stroke suggested by longitudinal studies of cortical physiology. Cereb Cortex. (2008) 18:1909-22. doi: 10.1093/cercor/bhm218

22. Thickbroom GW, Byrnes ML, Archer SA, Mastaglia FL. Motor outcome after subcortical stroke: MEPs correlate with hand strength but not dexterity. Clin Neurophysiol. (2002) 113:2025-9. doi: 10.1016/S1388-2457(02)00318-8

23. Talelli P, Greenwood RJ, Rothwell JC. Arm function after stroke: neurophysiological correlates and recovery mechanisms assessed by transcranial magnetic stimulation. Clin Neurophysiol. (2006) 117:1641-59. doi: 10.1016/j.clinph.2006.01.016

24. Devanne H, Lavoie BA, Capaday C. Input-output properties and gain changes in the human corticospinal pathway. Exp Brain Res. (1997) 114:32938. doi: 10.1007/PL00005641

25. Marcus HJ, Carpenter KL, Price SJ, Hutchinson PJ. In vivo assessment of high-grade glioma biochemistry using microdialysis: a study of energyrelated molecules, growth factors and cytokines. J Neurooncol. (2010) 97:1123. doi: 10.1007/s11060-009-9990-5

26. Sontheimer HJ. A role for glutamate in growth and invasion of primary brain tumors. Neurochem. (2008) 105:28795. doi: 10.1111/j.1471-4159.2008.05301.x

27. Tantillo E, Vannini E, Cerri C, Spalletti C, Colistra A, Mazzanti $\mathrm{CM}$, et al. Differential roles of pyramidal and fast-spiking, GABAergic neurons in the control of glioma cell proliferation. Neurobiol Dis. (2020) 141:104942. doi: 10.1016/j.nbd.2020.104942

28. Bowden JL, Taylor JL, McNulty PA. Voluntary activation is reduced in both the more-and less-affected upper limbs after unilateral stroke. Front Neurol. (2014) 5:239. doi: 10.3389/fneur.2014.00239

29. Di Lazzaro V, Restuccia D, Oliviero A, Profice P, Ferrara P, Insola A, et al. Magnetic transcranial stimulation at intensities below active motor threshold activates intracortical inhibitory circuits. Exp Brain Res. (1998) 119:2658. doi: 10.1007/s002210050341

30. Nakamura H, Kitagawa H, Kawaguchi Y, Tsuji H. Intracortical facilitation and inhibition after transcranial magnetic stimulation in conscious humans. J Physiol. (1997) 498:817-23. doi: 10.1113/jphysiol.1997.sp021905

31. Irlbacher $\mathrm{K}$, Brandt SA, Meyer BU. In vivo study indicating loss of intracortical inhibition in tumor-associated epilepsy. Ann Neurol. (2002) 52:119-22. doi: 10.1002/ana.10229

32. Honaga K, Fujiwara T, Tsuji T, Hase K, Ushiba J, Liu M. State of intracortical inhibitory interneuron activity in patients with chronic stroke. Clin Neurophysiol. (2013) 124:364-70. doi: 10.1016/j.clinph.2012.08.005

33. Lim L, Mi D, Llorca A, Marín O. Development and functional diversification of cortical interneurons. Neuron. (2018) 100:294313. doi: 10.1016/j.neuron.2018.10.009
34. Tewari BP, Chaunsali L, Campbell SL, Patel DC, Goode AE, Sontheimer H. Perineuronal nets decrease membrane capacitance of peritumoral fast spiking interneurons in a model of epilepsy. Nat Commun. (2018) 9:4724. doi: 10.1038/s41467-018-07113-0

35. Blanchart A, Fernando R, Häring M, Assaife-Lopes N, Romanov RA, Andäng $\mathrm{M}$, et al. Endogenous GABAA receptor activity suppresses glioma growth. Oncogene. (2017) 36:777-86. doi: 10.1038/onc.2016.245

36. Venkatesh HS, Johung TB, Caretti V, Noll A, Tang Y, Nagaraja S, et al. Neuronal activity promotes glioma growth through neuroligin-3 secretion. Cell. (2015) 161:803-16. doi: 10.1016/j.cell.2015.04.012

37. Di Lazzaro V, Oliviero A, Profice P, Pennisi,MA., Di Giovanni S, Zito G, et al. Muscarinic receptor blockade has differential effects on the excitability of intracortical circuits in the human motor cortex. Exp Brain Res. (2000) 135:455-61. doi: 10.1007/s002210000543

38. Turco CV, El-Sayes J, Savoie MJ, Fassett HJ, Locke MB, Nelson AJ. Short- and long-latency afferent inhibition; uses, mechanisms and influencing factors. Brain Stimul. (2018) 11:59-74. doi: 10.1016/j.brs.2017.09.009

39. Di Lazzaro V, Profice P, Pilato F, Capone F, Ranieri F, Florio L, et al. The level of cortical afferent inhibition in acute stroke correlates with long-term functional recovery in humans. Stroke. (2012) 43:2502. doi: 10.1161/STROKEAHA.111.631085

40. Otten ML, Mikell CB, Youngerman BE, Liston C, Sisti MB, Bruce $\mathrm{JN}$, et al. Motor deficits correlate with resting state motor network connectivity in patients with brain tumours. Brain. (2012) 135:101726. doi: 10.1093/brain/aws041

Conflict of Interest: The authors declare that the research was conducted in the absence of any commercial or financial relationships that could be construed as a potential conflict of interest.

Publisher's Note: All claims expressed in this article are solely those of the authors and do not necessarily represent those of their affiliated organizations, or those of the publisher, the editors and the reviewers. Any product that may be evaluated in this article, or claim that may be made by its manufacturer, is not guaranteed or endorsed by the publisher.

Copyright (c) 2021 Rizzo, Terranova, Raffa, Cardali, Angileri, Marzano, Quattropani, Germanò, Girlanda and Quartarone. This is an open-access article distributed under the terms of the Creative Commons Attribution License (CC BY). The use, distribution or reproduction in other forums is permitted, provided the original author(s) and the copyright owner(s) are credited and that the original publication in this journal is cited, in accordance with accepted academic practice. No use, distribution or reproduction is permitted which does not comply with these terms. 